\title{
Proliferative diabetic retinopathy
}

\section{Site and size of initial lesions}

\author{
ENID TAYLOR AND J. H. DOBREE \\ St. Bartholomew's Hospital, London
}

The pathogenesis of proliferative diabetic retinopathy is unknown. Although there are some established clinical and histological facts, the exact sequence of events is yet to be determined. Diabetic angiopathy is widespread in the body and appears to be a complication of and related to the duration of the diabetic state. The essential difference between this and proliferative diabetic retinopathy is the absence of microaneurysm formation and neovascularization outside the eye. It thus seems that local ocular factors, either genetic, metabolic, or anatomical, modify the generalized response. The role of the last factor has not yet been investigated, so that it is not known whether the anatomical pattern of the retinal arteries and veins has any relationship to the sites and size of areas of neovascularization.

It is the purpose of this paper to study the size and distribution of those areas in early proliferative diabetic retinopathy which are of sufficient size to be visible in fundus photographs and to subject them to statistical analysis to see if any definable pattern emerges.

\section{Material}

I03 patients with proliferative diabetic retinopathy are under regular review with regard to their diabetic state and in particular the fundus changes. I 70 eyes in these patients were studied. All fundi were photographed in multiple fields using the Zeiss fundus camera at about 3-monthly intervals. The data collected in this survey are taken from these photographic records made at the first or second visits.

Of the 170 eyes studied, 86 were suitable for inclusion in the present survey as the new vessels were in an early stage of formation. The remainder were excluded because the areas of proliferation were too diffuse and advanced at the initial visit and the origin of the new vessels was obscured or obliterated by connective tissue. The criteria for inclusion were:

(I) There were good photographic records of the fundus at the first or second visit.

(2) There was photographic continuity from the area of neovascularization to the optic disc, using one or more photographic fields.

(3) The site of origin of an area of neovascularization was discrete, so that the relationship of the feeding vessels to the main retinal vein and the optic disc could be accurately measured.

\section{Method}

A fixed projector was used to project the photographs a fixed distance onto a solid white wooden screen. All areas of neovascularization visible on the initial photographs were documented, thus giving a record of the lesions as they present to the ophthalmologist. A composite diagram was 
made for each eye to show two-dimensional relationships of the optic disc, retinal arteries and veins, and areas of neovascularization. The sites of the lesions were divided into three main groups:

(r) Arising from the optic disc.

(2) Isolated retinal lesions.

(3) Associated with a retinal vein.

The last group was further subdivided into the four main drainage areas, superior and inferior temporal and superior and inferior nasal.

The position of the lesion in the fundus was determined by the following measurements (Fig. 1):

(x) The distance along a vein from the disc margin to the origin of the feeding vessels (B).

(2) The distance along a vein from the disc margin to the arterio-venous crossing nearest to the lesion on the same vein $(\mathrm{C})$.

(3) The radial distance of the lesion from the centre of the optic disc to the centre of the lesion (D).

(4) The angle subtended by a lesion from the centre of the optic disc.

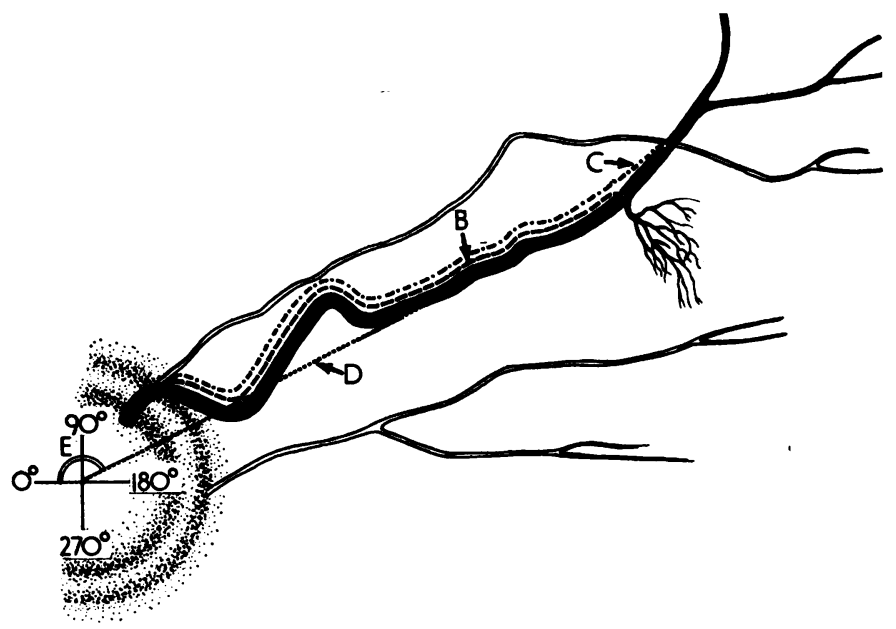

FIG. I Drawing of sector of right fundus, showing measurements made to determine the site of new vessel formation

(B) Distance along a vein from the disc margin to the site of a lesion

(C) Distance along a vein from the disc margin to the arterio-venous crossing nearest the lesion

(D) Radial distance from the centre of the optic disc to the centre of the lesion

(E) Angle

In the right eye, the angle was measured $0^{\circ}-360^{\circ}$ reading clockwise, $0^{\circ}$ being at $90^{\prime}$ clock on the fundus.

In the left eye a mirror image of the angle reading was made, thus $0^{\circ}$ lay at $30^{\prime}$ 'clock and was measured to $360^{\circ}$ in an anti-clockwise direction.

By this means, for example, all lesions in the upper temporal field, whether in the right or left eye, lay between $0^{\circ}$ and $90^{\circ}$, thus standardizing the results (see E in Fig. 1).

The linear measurements were made with an opisometer on the projected photograph on the wooden screen. The arbitrary figures so obtained were converted to disc diameters with reference to the opisometer measurement of the optic disc for the same eye.

A quantitative assessment was made of the size of the lesions. As the lesions are irregular in outline, the diameter varies in all directions, and therefore grouped estimates of these diameters were used.

Lesions were placed in four categories according to the diameter:

(1) Less than half a disc diameter.

(2) From half to one disc diameter.

(3) One to two disc diameters.

(4) Greater than two disc diameters. 


\section{Results}

A total of 248 new vessel formations were seen in the 86 eyes studied. Although the eye is a sphere, and the measurements have been made on a flat surface, the distortion is small and similar for all measurements, so that the comparison of the data is valid. The results are shown in Table $I^{*}$.

These data have been subjected to a statistical analysis in respect of $(a)$ distribution of lesions, (b) size of lesions, $(c)$ distance of lesions from optic disc, and $(d)$ relationship of lesions to arterio-venous crossings.

\section{(a) Distribution of lesions}

(I) GENERAL

There were 248 lesions in the 86 eyes; 63 (73.25 per cent.) showed lesions on the optic disc, and in 14 eyes the neovascularization was confined to this site.

The number of lesions associated with each of the four main venous drainage areas is shown in Table II. The number of isolated retinal lesions was very small, only nine out of 248. This group contained too few examples for any significant conclusions to be drawn, and it is therefore excluded from subsequent calculations.

Table II Distribution of 248 areas of neovascularization in 86 eyes

\begin{tabular}{lc}
\hline Site & $\begin{array}{c}\text { No. of } \\
\text { lesions }\end{array}$ \\
\hline Optic disc & 63 \\
\hline Upper temporal vein & 63 \\
\hline Lower temporal vein & 47 \\
\hline Lower nasal vein & 29 \\
\hline Upper nasal vein & 37 \\
\hline Isolated retinal & 9 \\
\hline
\end{tabular}

Table III Distribution of lesions by size

\begin{tabular}{|c|c|c|}
\hline $\begin{array}{l}\text { Size } \\
\text { (disc diameters) }\end{array}$ & $\begin{array}{l}\text { On } \\
\text { disc }\end{array}$ & $\begin{array}{l}\text { On retinal } \\
\text { veins }\end{array}$ \\
\hline$<\frac{1}{2}$ & 14 & 36 \\
\hline$\frac{1}{2}-1$ & 8 & 76 \\
\hline $1-2$ & 14 & $5^{2}$ \\
\hline$>2$ & 27 & 12 \\
\hline
\end{tabular}

(2) AMONGST RETINAL VEINS

I 76 lesions were distributed amongst the four main branches of the retinal veins (Table II). This distribution is uneven, most lesions occurring in association with the superior temporal veins and fewest with the inferior nasal veins. This difference is significant $\left(\chi^{2}=14.64\right.$; 3 d.f.; 0.0 I $>$ P $>0.005$ ).

These findings can be represented pictorially, using the radial measurements and the angles of the lesions (Fig. 2, overleaf); this demonstrates clearly the lesions lying along the paths of the main vascular branches, with the greatest concentration in the superior temporal field.

The lesions related to the nasal vessels lie nearer the optic disc. Of particular interest is the fact that there are no proliferative lesions in the macular and paramacular areas.

* Because Table I is very large and detailed it has been decided not to publish it with this paper. The data is available to anyone interested in this subject on application to the authors. 


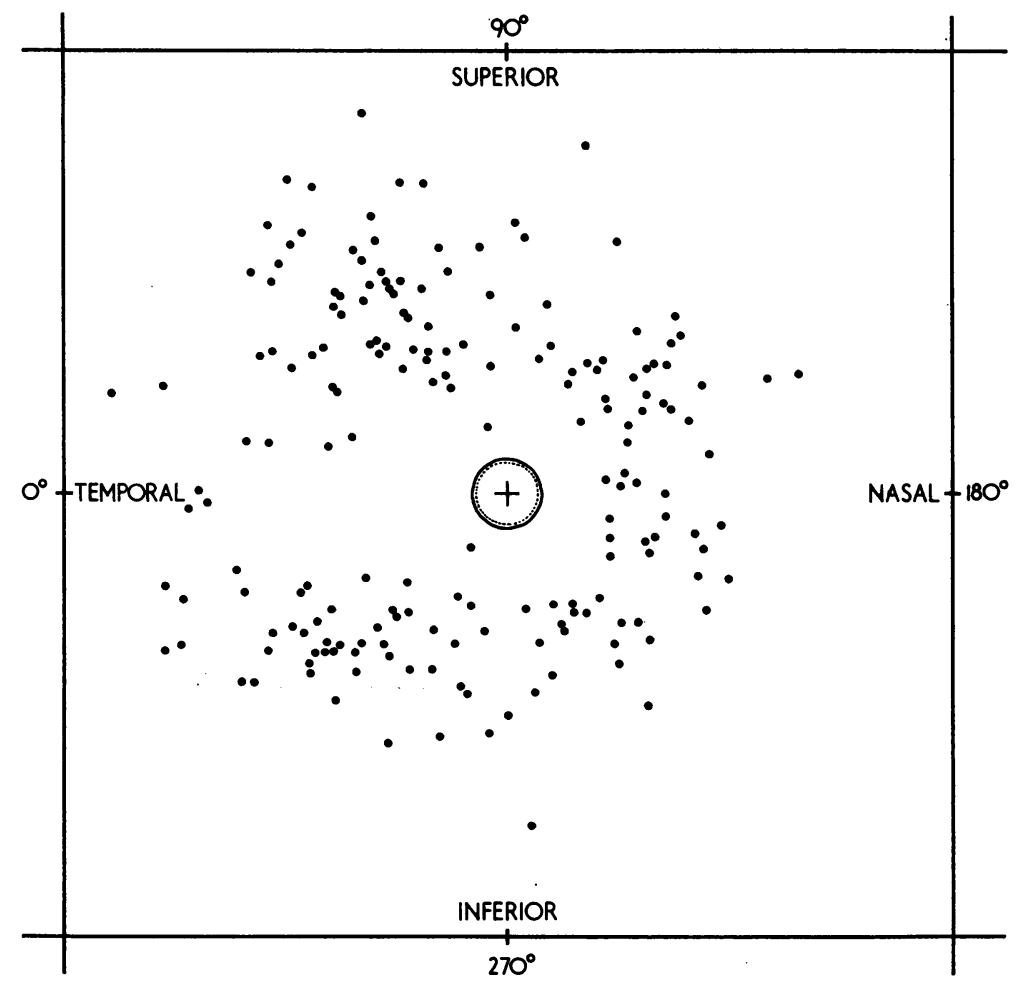

FIG. 2 Schematic representation of the right fundus, showing points of origin of 185 areas of neovascularization surrounding the optic disc. The lesions in the left eye have been transposed laterally in order to include them in the same Figure

\section{(b) Size of lesions}

The distribution of lesion size is shown in Table III. There were more of the larger lesions on the disc than in association with the retinal veins; thus 42.8 per cent. of disc lesions were greater than 2 disc diameters in size when first seen, whereas only 6.8 per cent. of the remaining lesions exceeded 2 disc diameters. These figures are highly significant $\left(\chi^{2}=\right.$ $49 \cdot 94 ; 3$ d.f.; $\mathrm{P} \ll 0 \cdot 001$ ).

The distribution of lesion sizes amongst the four main retinal veins is shown in Table IV. The findings are not significant $\left(\chi^{2}=10.35 ; 9\right.$ d.f.; $\left.0.4>\mathrm{P}>0.3\right)$. Thus there is no tendency for a lesion of a particular size to occur on a particular vein, but it is not possible to say whether this size scatter is truly random.

Table IV Distribution of lesion sizes by site on retinal veins

\begin{tabular}{|c|c|c|c|c|}
\hline \multirow{2}{*}{$\begin{array}{l}\text { Size } \\
\text { (disc diameters) }\end{array}$} & \multicolumn{2}{|c|}{ Temporal } & \multicolumn{2}{|l|}{ Nasal } \\
\hline & Superior & Inferior & Inferior & Superior \\
\hline$\frac{1}{2}$ & 8 & 13 & 8 & 7 \\
\hline$\frac{1}{2}-1$ & 29 & 19 & 12 & I6 \\
\hline$I-2$ & 18 & 13 & 9 & 12 \\
\hline 2 & 8 & 2 & o & 2 \\
\hline
\end{tabular}




\section{(c) Distance of lesions on the veins from the optic disc (Table V)}

There was no significant difference in mean distance from the optic disc margin to the origin of the lesion between the superior and inferior temporal veins or between the superior and inferior nasal veins. It seemed reasonable, therefore, to combine the data into temporal and nasal groups as in Table VI. The difference between the mean distances in the temporal and nasal fields is now highly significant (Student's test is 5.56 , 174 d.f.; $\mathrm{P} \ll 0 \cdot 00 \mathrm{I})$. Thus lesions associated with temporal venous branches tend to occur further from the disc margin than those associated with nasal venous branches.

Table $\mathbf{V}$ Site of lesions on veins, by distance from optic disc

\begin{tabular}{llllll}
\hline \multirow{2}{*}{ Site } & & $\begin{array}{l}\text { No. of } \\
\text { lesions }\end{array}$ & $\begin{array}{l}\text { Mean distance } \\
\text { along vein } \\
\text { from disc } \\
\text { (disc diameters) }\end{array}$ & Variance & $\begin{array}{l}\text { Standard } \\
\text { deviation }\end{array}$ \\
\hline Temporal & Superior & 63 & $3 \cdot 44$ & $\mathbf{1} \cdot 55$ & $\mathbf{1} \cdot 245$ \\
\cline { 2 - 7 } & Inferior & 47 & $3 \cdot 24$ & $\mathbf{1} \cdot 45$ & $\mathbf{1} \cdot 204$ \\
\hline Nasal & Inferior & 29 & $2 \cdot 2 \mathrm{I}$ & $\mathbf{0} \cdot 55$ & $\mathbf{0} \cdot 74 \mathbf{1}$ \\
\cline { 2 - 7 } & Superior & 37 & $2 \cdot 46$ & $\mathbf{0} \cdot 95$ & $\mathbf{0} \cdot 972$ \\
\hline
\end{tabular}

Table VI Site of lesions on veins related to distance from optic disc, temporal and nasal groups combined

\begin{tabular}{lllll}
\hline Site & $\begin{array}{l}\text { No. of } \\
\text { lesions }\end{array}$ & $\begin{array}{l}\text { Mean distance } \\
\text { along vein } \\
\text { from disc } \\
\text { (disc diameters) }\end{array}$ & Variance & $\begin{array}{l}\text { Standard } \\
\text { deviation }\end{array}$ \\
\hline Temporal & 1 10 & 3.36 & $1 \cdot 50$ & $1 \cdot 22$ \\
\hline Nasal & 66 & $2 \cdot 35$ & $0 \cdot 76$ & 0.87 \\
\hline
\end{tabular}

\section{(d) Distribution of lesions related to arterio-venous crossings}

The measurements used for this analysis are the distance from the margin of the optic disc to the lesion, and from the margin of the optic disc to the arterio-venous crossing nearest to the lesion. I 76 lesions were clearly associated with the retinal veins (see $a \mathrm{I}$ and $a 2$, above). Of these, I 29 have a crossing nearby on the same vein. On twelve occasions, two lesions were associated in pairs with the same arterio-venous crossing, making a further 24 lesions. There were only two instances in which three areas of neovascularization were grouped around a single arterio-venous crossing, making six lesions in all. The lesions lay on the disc side of the crossing in sixty cases, and peripheral to it in 99 cases. One other lesion has been excluded, as the only arterio-venous crossing was associated with a different branch of the same vein.

The remaining sixteen areas of neovascularization have no crossing on the vein at any point. All of these except one lie in the nasal field, six superior and nine inferior.

The I 59 lesions associated with an arterio-venous crossing have been used in the analysis. The distances from the optic disc to the lesion and from the optic disc to the arterio-venous crossing have been plotted as the co-ordinates of a graph (Fig. 3, overleaf). This shows 


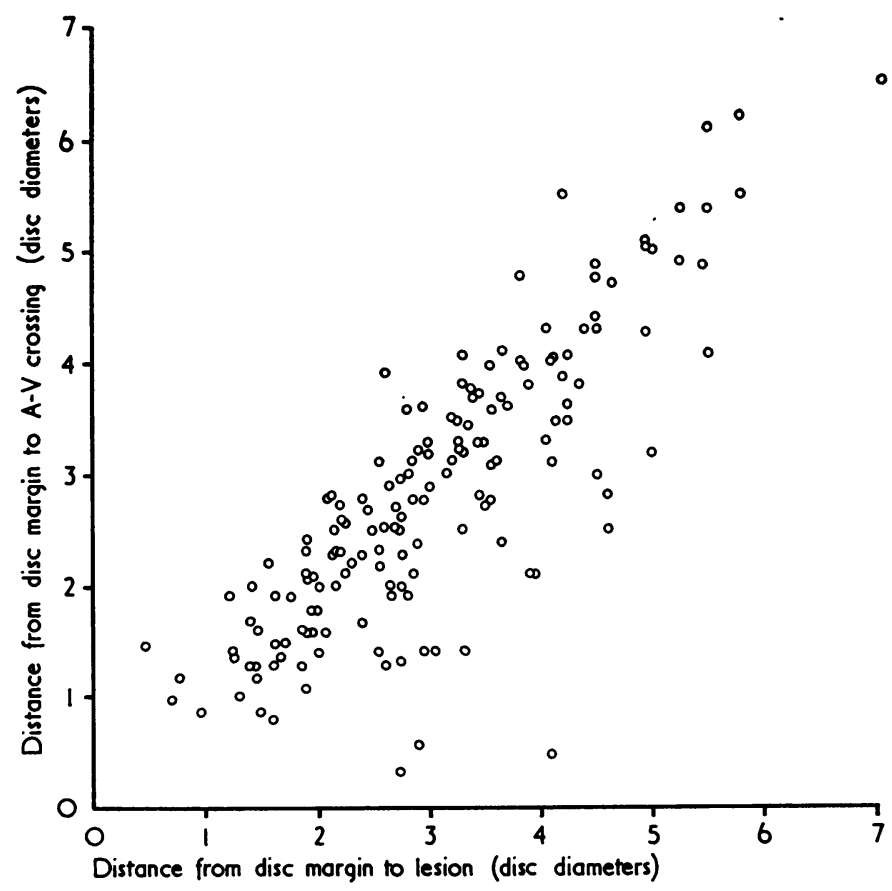

FIG. 3 Graph showing a high degree of correlation between site of lesion and nearest arteriovenous crossing

that the points fall close to a straight line, thus showing a high degree of correlation between the two measurements-i.e. the lesions are related to the crossings and are not therefore randomly distributed.

\section{Discussion}

Venous and capillary changes in proliferative diabetic retinopathy were originally thought to represent localized limited venous thromboses, and Bonnet and Bonamour (1938) described the retinal changes in several cases of diabetes mellitus to support this theory. They particularly noted neovascularization at an arterio-venous crossing in two cases. Ashton ( 1963 ) showed that the early changes in proliferative diabetic retinopathy of microaneurysm formation and neovascularization are seen histologically in many other systemic and ocular disorders. They should therefore be regarded as a response to similar stimuli (Ashton, 1967). He has further suggested that this stimulus is a vaso-formative factor released from localized areas of hypoxic retina. Wise (1956) had previously supported the existence of an unknown factor (Michaelson's X factor, I954), which stimulated new vessel formation, and he believed that it was produced in all cases of retinal neovascularization, and that moreover the underlying pathological state responsible for its production was venous obstruction.

In this anatomical survey, we have found that areas of neovascularization arise from the disc and from the main temporal and nasal veins. With regard to the venous lesions the following points were noted:

(I) Lesions occur more frequently on the temporal than on the nasal veins;

(2) Lesions are most commonly seen on the superior temporal veins;

(3) Temporal lesions lie farther from the disc margin than nasal lesions; 
(4) All the temporal lesions except one, and 80 per cent. of the nasal lesions, are associated with an arterio-venous crossing;

(5) A high degree of correlation exists between the lesion and the site of an arteriotvenous crossing.

Koyanagi (1928a, b) found a greater number of arterio-venous crossings associated with the superior temporal vein than in any other venous drainage area. It is therefore reasonable to postulate that the larger number of arterio-venous crossings in the superior temporal field predisposes to the larger number of lesions found in this venous drainage field.

Dobree (1964) reported that thirteen out of 25 eyes ( 52 per cent.) showed disc neovascularization at the initial visit, but in the present series the incidence is 73.25 per cent. Moreover, at the initial visit, disc lesions are larger than those arising from retinal veins.

As Fig. 2 demonstrates, proliferative lesions were absent over the posterior pole. This is in contrast to the angiopathy found in a recent study of exudative retinopathy by Dobree (1970). In this it was found that the foci of pathological capillaries in exudative diabetic retinopathy lie preferentially at the posterior pole, especially lateral to the macula. This also confirms our clinical finding that exudative and proliferative lesions do not overlap anatomically.

An analysis of our results indicates that the development of lesions in proliferative diabetic retinopathy does not occur at random. The distribution of the site and size of disc and retinal lesions show distinctive features, and a positive correlation exists between the lesions associated with the retinal veins and the arterio-venous crossings. Although in this series no subdivision of lesion site on the optic disc has been made, it may be relevant that there are arterio-venous crossings on all discs. The answer to the question whether these results can be grouped under one aetiological factor awaits further study. The most likely supposition is that this factor is of a mechanical nature, such as a localized disturbance in haemodynamics at the arterio-venous crossing, thus affecting the blood flow and oxygenation in a localized area of the retina. Alternatively, it may be a purely mechanical obstruction to blood flow, and the areas of neovascularization are primarily anastomotic in nature. In either case, it is the mechanical factor leading to localized metabolic disturbance within the retina which determines the site of the initial lesion.

\section{Summary}

The initial photographs of 86 eyes with proliferative diabetic retinopathy have been studied. A statistical survey has been carried out with respect to the site and size of 248 areas of neovascularization. The following observations were made:

(I) 73.25 per cent. of eyes have areas of new vessel formation on the optic disc and these areas are larger than those associated with retinal veins.

(2) Lesions associated with retinal veins show the following characteristics:

(a) They occur most frequently on the superior temporal veins,

(b) Temporal lesions lie farther from the optic disc than nasal lesions,

(c) A high degree of correlation exists between the site of a lesion and the nearest arteriovenous crossing.

We wish to thank Dr. Alan Jennings for assistance with the statistical analysis and Miss Christine Otway for secretarial help. 


\section{References}

Ashton, N. (1963) Brit. 7. Ophthal., 47, 521

(1967) In "Vascular Complications of Diabetes Mellitus", ed. S. J. Kimura and W. M.

Caygill, p. 3. Mosby, St. Louis

Bonnet, P., and вonamour, G. (1938) Bull. Soc. Ophtal. Paris, 50, 367

DOBREe, J. H. (1964) Erit. F. Ophthal., 48, 637

$$
\text { (1970) Ibid., 54, I }
$$

koyanagi, y. (1928a, b) Klin. Mbl. Augenheilk., 80, 436; 81, 2 I 9

michaelson, i. c. (1954) "Retinal Circulation in Man and Animals", p. i 18. Thomas, Springfield,

Ill.

wise, G. N. (1956) Trans. Amer. ophthal. Soc., 54, 729 